\title{
Sequential priming in visual search: Contributions of stimulus-driven facilitation and learned expectancies
}

\author{
PATRICIA M. BLOUGH and DONNA M. LACOURSE \\ Brown University, Providence, Rhode Island
}

\begin{abstract}
Sequential priming refers to speeded visual search when target identity or location is repeated within a trial sequence. In two experiments with pigeons, we addressed the relative contributions of stimulus-driven factors and learned expectancies to this effect. Pigeons pecked at targets during trialwise presentations of visual-search displays. Random-sequence conditions minimized the role of expectancy by introducing same-target or same-location trial sequences unpredictably. Blocked-sequence conditions added predictability by regular repetition of target and/or location over trials. Intertrial interval varied from 0.5 to $3 \mathrm{sec}$. The findings revealed significant reductions in reaction time during predictable target or location sequences compared with unpredictable repetitions within random contexts. Stimulus-driven factors do not seem to have an important role in many instances of sequential priming. Expectancy-based priming of target and location followed similar patterns.
\end{abstract}

Foraging birds may experience several food types within a patch. Converging evidence indicates that repeated encounters with a given prey facilitate its detection. Tinbergen's (1960) extensive field studies provided early documentation of this phenomenon, which he attributed to the formation of a searching image. Recent laboratory studies have replicated and further analyzed the repetition effect under a variety of conditions. For example, Bond (1983) and Reid and Shettleworth (1992) provided opportunities for free foraging among grains. Other work employed brief search trials requiring the detection of photographically displayed food (Bond \& Riley, 1991) or alphanumeric targets in computer displays (P. M. Blough, 1989, 1991, 1992). In these studies, repetition was explored either by varying the within-session probability of a given target and thus the likelihood that the encounter would be repeated (e.g., Bond, 1983; P. M. Blough, 1992) or by withinsession comparisons between same-target and mixedtarget trial blocks (e.g., P. M. Blough, 1989, 1991; Bond \& Riley, 1991: Pietrewicz \& Kamil, 1979).

Although Tinbergen (1960) proposed an attentional account of the repeated-encounters effect, he and others noted alternative explanations - many of which have been ruled out by the subsequent work. For example, birds do more than improve their prey-handling techniques, because the effect is seen in well-practiced sub-

This research was supported by Grant BNS 88-19876 from the National Science Foundation. We are grateful to Donald S. Blough for his comments and suggestions. Address correspondence to P. M. Blough, Department of Psychology, Brown University, Providence, RI 02912. jects with simple response measures (e.g., P. M. Blough, 1989; Pietrewicz \& Kamil, 1979). Nor do birds simply adopt more efficient travel paths, because, for example, in Blough's designs, the target's position varied randomly. Finally, recent data (P. M. Blough, 1992; Reid \& Shettleworth, 1992, Experiment 3) are not consistent with the suggestion that enhanced detection results simply from adjustments of search speed to prey discriminability (Guilford \& Dawkins, 1987). P. M. Blough $(1989,1991)$ suggested that a sequence of encounters with the same target acts as an attentional priming operation; that is, a representation of the target is activated in advance of a search opportunity and thus facilitates detection. The effects of sequential priming can be enduring; in our 1991 study they persisted over intertrial intervals lasting as long as $17 \mathrm{sec}$, and in Tinbergen's field studies, lengthy periods intervened between prey capture and the bird's return to the foraging area.

In the present work, we explore the sources of such activation. Previously (P. M. Blough, 1989) we invoked the informational value of single-target sequences, noting parallels to human priming studies that have shown faster responses for predictable targets (e.g., Beller, 1971). We use the word expectancy to refer to a learned association between an advance cue and a particular target property. Pretrial reference to this association would permit activation of that property.

Although expectancy seems to contribute to priming, unlearned influences could constitute a second source of activation. For example, a neural trace induced by the preceding target might extend its activation to the next search opportunity. Unlearned or stimulus-driven facil- 
itation depends on local trial interactions and is best identified under conditions that rule out expectancy effects. Research with humans meets that requirement. For example, a letter prime expedites identification of its matching target letter, even when the prime is an invalid predictor of the subsequent target's identity (Posner \& Snyder, 1975). In a sequence of choice trials, quick repetition of a previous trial leads to faster detection within random sequences (Kirby, 1976, 1980). The duration of such effects appears to be relatively short, perhaps because the mediating trace fades quickly.

Unfortunately, the animal research cited above does not distinguish between stimulus-driven and expectancybased sources of priming, because the predictability of the forthcoming target has always been confounded with local target repetition. In the present study, we explored stimulus-driven and expectancy-based priming of target location as well as target identity. Numerous human studies have stressed the facilitating effects on search of cues predicting target position. Effective cues include pretrial information provided by the relative frequency with which a target appears in a particular region of the display (Shaw \& Shaw, 1977). With pigeons, D. S. Blough (1993) showed that within-display cues to general target location expedited target detection. However, we do not know of studies in which location cuing has been explored by trial repetition in animal visual search.

In the present experiments, we used trialwise visualsearch procedures, in which pigeons detected a single target displayed amidst multiple distractors on a video monitor. To isolate stimulus-driven sources of priming, we compared conditions in which the identity and/or location of the forthcoming target was and was not predictable. In Experiment 1, we explored target and location repetition at several intertrial intervals (ITIs). During Phase 1, pseudorandom target and location sequences included infrequent and unpredictable repetitions, thus minimizing information about forthcoming trials. Sequential priming within this context would be restricted to local trial interactions and attributable to stimulus-driven factors. During Phase 2, blocked sametarget and/or same-location sequences provided cues to the forthcoming target's identity, location, or both, permitting an expectancy-based source of priming. In Experiment 2, we replicated a portion of this work, adding new controls.

We hypothesized that (1) reaction times (RTs) within blocked sequences during Phase 2 would be lower than those within nonrepeated trial sequences during Phase 1; (2) any stimulus-driven priming by same-target or samelocation sequences within Phase 1 would be seen as reduced RTs, as compared with nonrepeated trial sequences within that phase; (3) priming effects during Phase 1 would be no greater than those during Phase 2, because the blocked condition permitted facilitating influences of both stimulus-driven influences and expectancy.

\section{EXPERIMENT 1}

\section{Method}

\section{Subjects}

The subjects were 5 male White Carneaux pigeons. Group 1 consisted of three experimentally naive birds. The 2 birds in Group 2 had participated in other search experiments using letter targets. The birds were maintained at $80 \%$ of their ad-lib weights, and they typically collected their daily food ration during experimental sessions. They were housed in a pigeon colony room, where they had free access to water with added vitamins. A 17:7-h light:dark cycle was used.

\section{Apparatus}

Three experimental boxes were located in a separate room that was adjacent to a room containing the controlling equipment. Fans ventilated the boxes, and a loudspeaker supplied masking noise. Each box contained a subject chamber that was $30 \mathrm{~cm}$ wide $X$ $34 \mathrm{~cm}$ deep $\times 34 \mathrm{~cm}$ high. The chamber's front panel included two horizontally centered openings. One aperture, centered $7 \mathrm{~cm}$ above the floor, was $5 \mathrm{~cm}$ wide $\times 4 \mathrm{~cm}$ high and provided access to a food tray when raised. A dim lamp was mounted above the food tray. The second opening, centered $18 \mathrm{~cm}$ above the floor, was $12 \mathrm{~cm}$ wide $\times 8.5 \mathrm{~cm}$ high and provided access to a monochromatic video screen, $10.5 \mathrm{~cm}$ wide $\times 6.7 \mathrm{~cm}$ high. The screen was recessed by $3.2 \mathrm{~cm}$ and was surrounded by a Carroll touch frame. Programming software used the input from the touch frame to separate the screen into six equally wide vertical segments for the purpose of localizing keypecks. Controlling equipment included interfacing circuitry and IBM XT computers, which were used for sensing responses, controlling experimental events, and collecting data.

\section{Procedure}

As noted previously, the overall scheme was to compare a condition that included information about the forthcoming trial with a condition that minimized such information. In the informative condition (Phase 2), sessions were organized into successive blocks of trials within which the target and/or its location was repeated. In the noninformative condition (Phase 1), repeated-trial blocks were inserted infrequently and at random into sessions that otherwise selected targets and locations for each trial by a random method. We used a rather large number (11) of targets overall, because previous work had suggested that sequential priming is more robust with larger target sets (P. M. Blough, 1991). It was also important that the lengths of the planned sequences did not exceed the lengths of those that could occasionally occur by chance. Thus, the length of the inserted sequences was limited to two or three trials. For appropriate comparison, block size during the informative condition was three trials.

Stimulus displays. Test displays presented a single target among 23 distractors; the purpose of this complexity was to enhance the priming effect, seen best when targets are concealed (P. M. Blough, 1992). Display items were black characters on a light background. The distractors were an ampersand (\&) and the target drawn from a set of 11 letters (A, D, E, H, J, K, L, Q, S, U, $\mathrm{Y})$. These letters were selected for their relatively good discriminabilities from each other (D. S. Blough, 1985). The characters were $4 \mathrm{~mm}$ high and were arrayed in four slightly irregular rows and six vertically aligned columns, placed so that the separate columns were displayed in the centers of the screen's responselocalizing segments. Columns were separated by $11 \mathrm{~mm}$, and rows were separated by an average of $13 \mathrm{~mm}$.

Response contingencies. Trials were separated by ITIs, during which the screen had the gray value that was used for the background during trials. A momentary darkening occurred just before the new test display appeared. A response was defined as an un- 
interrupted sequence of two pecks to a given screen segment, with the exception noted below. A response to the segment containing the target was designated correct. On most trials, this was followed by the removal of the characters and a $0.5-\mathrm{sec}$ white band displayed at the bottom of the screen. The ITI then began. However, with a probability of .05 , a correct response produced the white band and mixed-grain reinforcement for a duration that maintained the bird's running weight within the experimental session. Following incorrect responses, the characters were removed, and a 2 -sec time-out was added to the ITI. The trial was then rerun until a correct response occurred. Such rerun trials were not included in the data or trial counts.

Training. The birds learned to peck at the target letters through autoshaping and, in some cases, handshaping methods. In subsequent training we used the trialwise program, gradually reducing the probability of food reinforcement and adding increasing numbers of distractors; training continued until the bird's accuracy stabilized.

Experimental conditions. There were two principal phases, which differed in the predictability of target identity and/or location. In Phase 1, target letters and locations were selected by a random-block method on baseline trials. Target and location blocks were made up of the 11 target letters and the six screen segments, respectively. Then one of the four locations within the selected screen segment was randomly chosen. In an additional subprogram, we added planned sets of two or three trials on which the target's identity, its location, or both would be identical within the set. The decision to enter this subprogram was made at random, with a probability of .17. Once entered, a random-block method was used to determine whether there should be two or three trials within the set and whether target, location, or both should be repeated. Altogether, there were six conditions within planned-sequence trials: 2 sequence lengths $\times 3$ sequence types. Food reinforcement was canceled between trials within the planned sets, and these sequences were terminated following errors.

Within Phase 1, there were four ITI values, each presented in a separate block of nine sessions. For Group 1, the ITI during the first block was $1 \mathrm{sec}$; in subsequent blocks, ITI was $3 \mathrm{sec}, 0.5 \mathrm{sec}$, and $2 \mathrm{sec}$, in that order. For Group 2, ITI order was 1, 2, 3, and $.5 \mathrm{sec}$. Data from the first session of each block were not counted. Sessions consisted of 1,200 recorded trials preceded by 12 warm-up trials.

In Phase 2, regular three-trial sequences of targets or locations were presented. In the initial condition, target identity was repeated within sequences of three trials. Each sequence began with a 2-sec warning display, made up of two centrally located columns that were $14 \mathrm{~mm}$ apart and composed of 17 closely spaced asterisks. A 1-sec ITI followed the cue's offset, which was followed by three trials. In these three trials, the chosen target was presented in a location that was redetermined on each trial by the randomblock program used in Phase 1. After each sequence, the target's identity was redetermined by a random-block method. In a second condition, the target's exact location was repeated in a similar manner. Within a 3-trial sequence, the target's position was unchanged, but its identity was redetermined on each trial. Dashes replaced the asterisks in the warning display. In a third condition, both target and location were repeated within the three-trial sequence. The warning cue consisted of carets $\left({ }^{\wedge}\right)$.

For the conditions evaluating target and location repetition separately, blocks of sessions were associated with each ITI by using the values and order of Phase 1 . For the condition evaluating target + location repetitions, there was a single block of sessions at the 1 -sec ITI. The first session in each block was not counted. Sessions in which accuracy was below $80 \%$ were also not counted, but these were rare. Additional sessions continued until median RTs associated with each sequence position met a four-session crite- rion for trend (P. M. Blough, 1984). Six additional sessions followed, for final data collection.

At the start of Phase 2, the number of pecks defining a response for Bird 374 was increased from 2 to 3 . The purpose of this change was to improve accuracy, which dropped from $87 \%$ to $77 \%$ at the start of this phase. Accuracy remained at Phase 1 levels for the remaining subjects.

\section{Results}

Overall accuracy was high; individual means ranged from $87 \%$ to $98 \%$ correct. These scores were not sensitive to variations in sequence type or ITI, and we do not describe them further. The primary data were withinsession median RTs for each position in a three-trial sequence. The data analysis applied to Phase 1 isolated baseline sequences of three trials, in which the target and location were different on each trial. The analysis also isolated three-trial sequences that repeated target and/or location, including any from unplanned as well as planned sets. All Phase 2 sequences repeated targets and/or locations. For both phases, the analysis applied only to sequences uninterrupted by errors or food reinforcement.

Figure 1 summarizes the findings. The points associated with Trials 1, 2, and 3 during Phase 1 are connected, because events did not set off sequences in any way. In contrast, during Phase 2, Trial 1 of each sequence followed the warning display. Because this event differed from events preceding Trials 2 and 3 , the points associated with the first trial are isolated. Reaction times associated with Trials 2 and 3 were compared by $t$ tests and did not differ significantly, and summary values averaged RTs for those trials. Within-subjects analyses of variance (ANOVAs) were used for statistical evaluation, and standard and protected $t$ tests for paired comparisons (Collyer \& Enns, 1987). A one-tailed criterion was applied to evaluations of sequence type, because of the directional nature of the experimental hypotheses. The significance criterion was set at $p \leq .05$.

The top row of Figure 1 shows RTs for target sequences at each of the four ITIs. The open symbols indicate data taken from Phase 1 in which the majority of sequences were random. Reaction times for the sametarget sequences (RAND/ST) did not decline over trials; indeed, those RTs as well as RTs for the baseline sequences (RAND/DTDL) overlapped considerably. For Phase $2(\mathrm{BLK} / \mathrm{ST})$, RTs associated with Trial 1 were relatively high, perhaps because of the prior warning signal. Reaction times associated with Trials 2 and 3 tended to be lower than those associated with Phase 1, but the difference diminished with increasing ITI. For reasons noted below, we submitted the effect of sequence type at the 3-sec ITI condition to an ANOVA. The outcome was not significant.

The means shown in Figure 1 represent well the data for 4 of the 5 birds. Bird 374 was exceptional in that its RTs were sharply raised throughout all conditions of Phase 2. This elevation could be attributable to the in- 

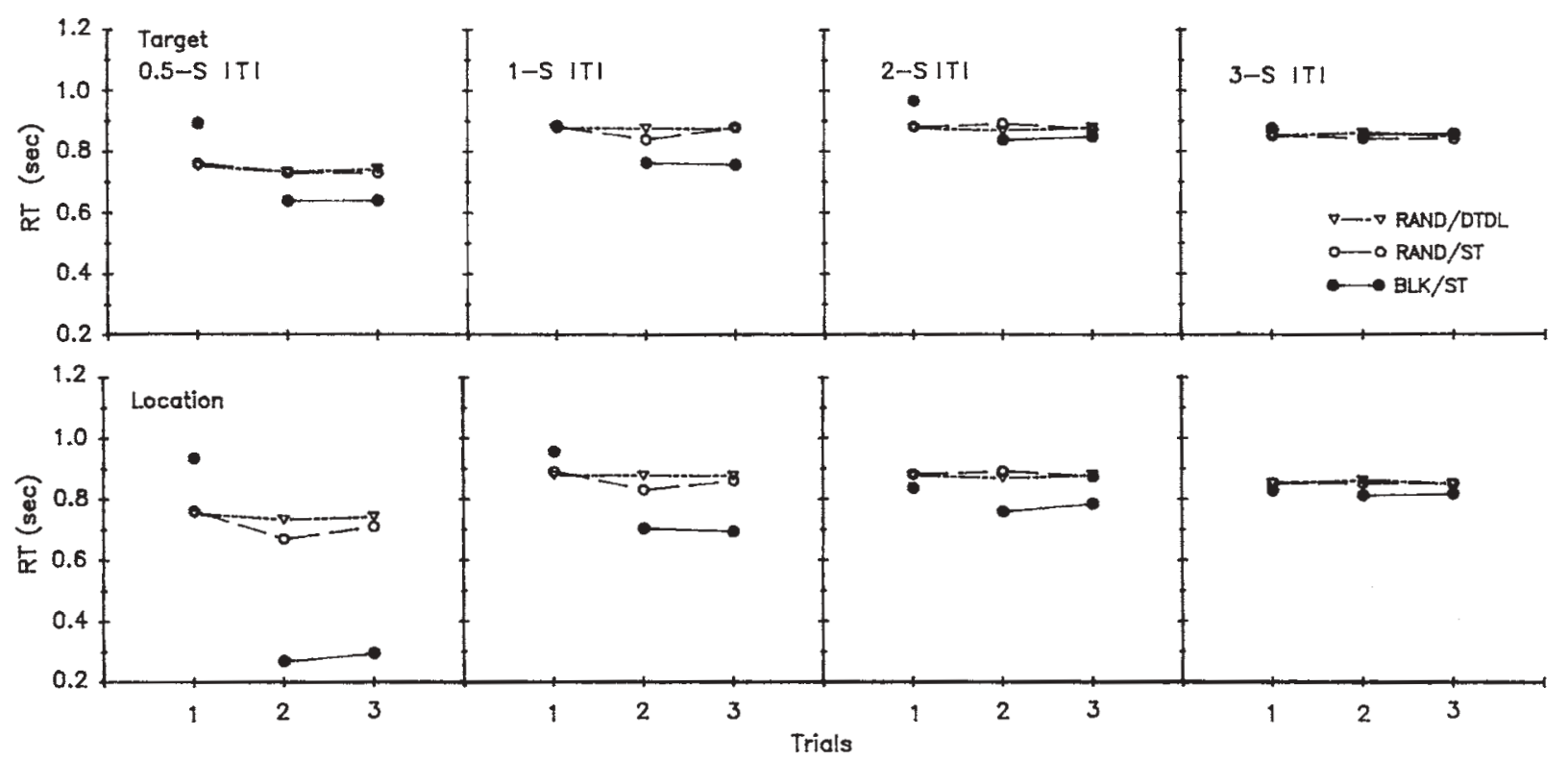

Figure 1. Top row: Reaction times (RTs) over three-target sequences in which target identity and location differed on each trial (RAND/DTDL), was repeated during occasional planned sets within random phase (RAND/ST), or was repeated consistently during blocked sets (BLK/ST). Data are shown for four intertrial intervals (ITIs). Bottom row: Data are presented as above, but the target's location was either repeated or not repeated within trial sequences.

crease in this bird's response requirement. To reduce the contribution of such extraneous variables, we adopted the 3-sec ITI condition as a normalization standard. The resulting individual scores expressed summary reaction time (RT) over Trials 2 and 3 at each ITI, divided by the summary RT for the comparable sequence type at the 3sec ITI.

An ANOVA was used to evaluate the corrected scores for effects of sequence type and ITI. The effects of sequence type $[F(2,8)=4.80]$ and ITI $[F(2,8)=11.87]$ were significant. There was no significant interaction between these factors. (Recall that the 3-sec ITI could not be used in the analysis, because it was the basis for normalization.) Protected $t$ tests were conducted to compare the three sequence types. There were no significant differences between baseline and RAND/ST sequences at any ITI, and there were no significant differences between the other sequence types at the 2-sec ITI. The difference between baseline and BLK/ST was significant at both .5 - and 1 -sec ITIs $[t(32)=2.06$ and 2.30 , respectively]. The difference between RAND/ST and BLK/ST was also significant at the two shorter ITIs $[t(32)=2.13$ and 2.30]. Thus, target repetition led to faster detection, but only when ITIs were relatively short and when the experimental context provided information about the forthcoming target's identity.

The bottom row of Figure 1 shows the data for the location sequences. Again, there was considerable overlap among scores within the random conditions, and these RTs dropped during the blocked condition, especially at the shorter ITIs. An ANOVA indicated that the effect of sequence type at the 3 -sec ITI was not significant. Thus, as before, the RTs averaged over Trials 2 and 3 were normalized with respect to this condition.

An ANOVA was used to evaluate the corrected scores for the effects of sequence type and ITI. The effects of sequence type $[F(2,8)=8.50]$ and ITI $[F(2,8)=36.90]$ were significant. The interaction between these factors was also significant $[F(4,16)=14.74]$. Protected $t$ tests were conducted to compare the three sequence types. There were no significant differences between baseline and RAND/SL sequences at any ITI, and there were no significant differences between the other sequence types at the 2-sec ITI. The difference between baseline and BLK/SL was significant at both .5- and 1-sec ITIs $[t(32)=6.10$ and 2.09, respectively $]$. The difference between RAND/SL and BLK/SL was significant only at the 0.5 -sec ITI $[t(32)=2.30]$ and approached significance at the $1-\sec$ ITI $[t(32)=1.66, .1>p>.05]$. As with target repetition, location repetition led to faster detection, but only when ITIs were relatively short and the experimental context provided information about the forthcoming target's location.

Figure 2 shows data for sequences in which both target and location were repeated. The blocked-sequence tests were conducted only at the 1-sec ITI, and it was not possible to apply the correction for the marked rise in Bird 374's RTs. Because there was a likely extraexperimental source for that elevation, we omitted Bird 374's data from the target + location analysis. In the left panel of Figure 2, sequence types are compared, as in Figure 1. Mean median RTs over Trials 2 and 3 were submitted to an ANOVA, which revealed a significant sequence-type effect $[F(2,6)=7.90]$. Protected $t$ tests showed signifi- 


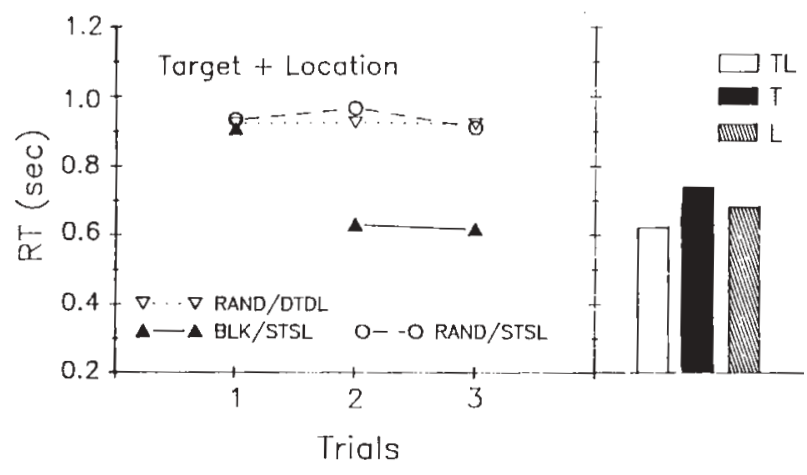

Figure 2. Outcome from target and location repetition (STSL) or nonrepetition (DTDL). Left panel shows comparison between random and blocked conditions, as in Figure 1. Right panel shows reaction times (RTs) averaged over Trials 2 and 3 of a sequence for blocked target (T), location(L), and target + location (TL) sequences.

cant reductions in RTs for the BLK/STL condition compared with the baseline and RAND/STL conditions $[t(6)$ $=3.35$ and $t(6)=3.53$, respectively]. The two random conditions were not significantly different. Thus, sequences repeating both target and location also led to faster target detection when such sequences were predictable.

The right panel of Figure 2 shows a comparison of summary RTs among the three types of blocked sequence at 1 -sec ITI. Protected $t$ tests for the comparisons did not reveal any significant effects. Thus, there was no indication of differential effects associated with target and location repetition and no advantage for their redundancy.

Overall, the results of Experiment 1 suggest that expectancy, but not stimulus-driven factors, influenced sequential priming. It is possible that order effects contributed to some of the RT differences; that is, blocked sequences always occurred in the second of the two experimental phases, and RT might have decreased with practice. Order cannot account for all of the effects in Experiment 1; the RT difference associated with the two phases disappeared at the 3-sec ITI, a condition that occurred midway during the course of Phase 1 . Nonetheless, a conceptual replication seemed desirable.

\section{EXPERIMENT 2}

Experiment 2 provided a stronger test of targetrepetition effects. Because predictable sequences occurred in the first of the two phases, training effects would not likely account for elevated RTs during subsequent unpredictable sequences. In addition, the design permitted a measure that would be less influenced by long-term RT drifts. Finally, the use of a more difficult target-distractor discrimination was intended to enhance priming effects (P. M. Blough, 1992). In this experiment, target repetition was explored at the 1-sec ITI.

\begin{abstract}
Method

\section{Subjects and Apparatus}

The birds and equipment were the same as those used in Experiment 1.

\section{Procedure}

Stimulus displays. Displays were organized like those in Experiment 1 . The target set was unchanged. However, in place of the homogeneous distractor set, distractors were drawn from a heterogeneous set of six characters $(1,7, \%, \&, \#, ?)$. This set of items was repeated four times across the screen; within-set order varied between sessions. Warning displays were made up of a horizontal row of characters (asterisks in Phase 1 and parentheses in Phase 2). The row extended across the two central screen segments midway between the top and bottom.
\end{abstract}

Response contingencies. In most respects, response contingencies were the same as those in Experiment 1. For Bird 073 the number of pecks defining a response was increased to three. The more important change was a response requirement to the warning display; the response, as defined previously, initiated the ITI when it was directed toward any portion of the warning cue.

Training. Sessions began with small display sizes, which increased gradually until accuracy was stable at the 24-item condition.

Experimental conditions. Sessions consisted of 1,200 trials made up of three-trial sequences. The ITI was constant at $1 \mathrm{sec}$. In Phase 1, a sequence presented the same target in variable locations; the warning cue preceded each sequence. This phase lasted until the bird completed 16 sessions. The final 4 sessions were used for analysis.

Following Phase 1, the birds worked 4 to 6 days on an interim condition in which targets were selected randomly on each trial. The new warning signal (parentheses) appeared before each set of three trials. This condition introduced the new warning cue and lasted until the birds met the stability criterion.

In the second phase, $80 \%$ of the sequences determined target identity by a random-block method. Of the remaining sequences, $10 \%$ presented the same target on each trial. The other $10 \%$ presented the same target on the first two trials and a new target on the third. Thus, the repetition of a target on the second trial did not forecast its repetition on the third. The decision to present a sametarget sequence was random, and a random-block method determined whether there should be two or three target repetitions within the same-target sequence. Food reinforcement was canceled between trials within these planned sets, and they were terminated following errors. This phase lasted for 21 sessions, and data from the final 20 were used for analysis.

\section{Results}

Mean accuracies for individual birds ranged from $88 \%$ to $98 \%$ correct and were insensitive to sequence type. Reaction time analysis applied to Phase 1 omitted three-trial sets interrupted by food reinforcement or errors. Analysis applied to Phase 2 isolated baseline sequences in which target identity differed over trials from sequences made up of three target repetitions. The data are summarized in Figure 3. Note that the comparable use of warning displays in the two phases permits consideration of all three within-sequence trials. During Phase 2 there was an overall elevation in RT, seen in the data for 4 of the 5 birds. Because the rise occurred in the first trial following the warning cue, nonexperimental variables may have contributed. Therefore, statistical analysis addressed only the slopes of the functions, cal- 


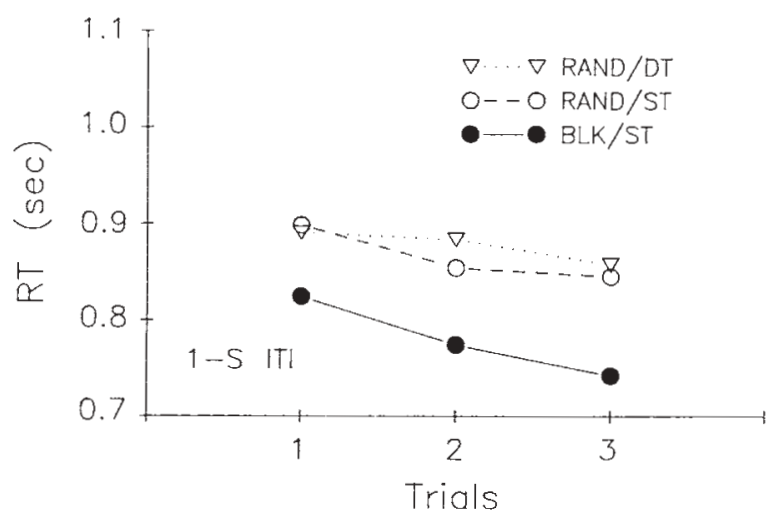

Figure 3. Data from Experiment 2, designed to compare slopes of the three functions. Data are organized as in Figure 1. Note, however, that baseline sequences (RAND/DT) could repeat target location, but not target identity.

culated as the percent change in RT between Trials 1 and 3.

A within-subjects ANOVA evaluated the effect of sequence type; the outcome just missed significance $[F(2,8)=3.74, .1>p>.05]$. Further comparisons used protected $t$ tests, appropriate in this case because they evaluated a priori hypotheses (Collyer \& Enns, 1987). The slope of the function associated with RAND/ST was not significantly greater than that associated with baseline (RAND/DT). However, the slope of the BLK/ST function was significantly steeper than that associated with baseline $[t(8)=2.65]$ and that associated with RAND/ST $[t(8)=1.91]$. Thus, the results of Experiment 2 indicated that target detection was more rapid when its identity was predictable. These findings confirm, in principle, those of Experiment 1.

\section{DISCUSSION}

Overall, this study showed no evidence of a stimulusdriven contribution to priming. During random phases, RTs were close in value and followed similar patterns within repeated and nonrepeated sequences; thus, there was no evidence for local effects between trials. During the blocked phase, however, predictable trial repetition yielded significant reductions in RT.

Stimulus-driven priming is relatively transient, and it could be argued that its effects decayed even within our shortest (1-sec) ITI. In his discussion, Kirby (1980) noted that, although preceding-trial effects persisted for less than $1 \mathrm{sec}$ when there were only two stimuli and two responses, they lasted $2 \mathrm{sec}$ or more when there were eight stimuli and eight responses. Kirby suggested that, in the two-choice paradigm, longer ITIs favored competing tendencies to guess the identity of the forthcoming target. The present work approximated the multiplechoice design, because several stimuli and response locations were used. Further, pigeons probably do not bring hypotheses about event sequences to the experimental chamber. However, we cannot rule out the possibility that the present study missed extremely brief facilitory effects.

Although this study established a clear contribution of expectancy to sequential priming, the effects diminished with ITI and disappeared at $3 \mathrm{sec}$. In contrast, P. M. Blough (1991) found that priming by predictable sequences survived ITIs up to $17 \mathrm{sec}$. In the earlier study, sequences were considerably longer (20 trials). In the present experiment, we used a larger target set (11 instead of 6) and considerably shorter sequences. These changes may have placed a heavier burden on memory for the preceding trial.

There also was no evidence for stimulus-driven priming of location; indeed, the pattern of results was similar to those for target repetition. Because the birds responded directly to the target, a bias to repeat the previous response would have appeared as a facilitating effect of same-location sequences. With the exception noted below, there was no evidence for such a bias. The birds had had considerable experience with search procedures that varied target locations; thus, they may have learned to switch among screen segments.

Location priming is well documented in human studies when advance cues indicate target position (Eriksen \& Hoffman, 1972) or when targets are more likely to appear in some locations than in others (Shaw \& Shaw, 1977). The blocked-location sequences reveal a related form of priming in pigeons and highlight the role of expectancy. We should note that, when correct location was predictable, attention to target features was unnecessary. Control by location alone may have accounted for the marked reduction in RT during the 0.5 -sec condition. With such brief separations between trials, a sequence approximated a free-operant condition. At the longer ITIs, decreased search speed may have reflected the resumption of target search.

It has been argued that location has a special status in perception (e.g., Tsal \& Lavie, 1993). The present findings did not indicate that location primes were more effective than identity primes except at the shortest ITI. The data also did not replicate human and pigeon studies showing greater facilitation when cues predicted both target and location (D. S. Blough, 1993; Lambert \& Hockey, 1986; Miller, 1988). It should be noted that the methods varied considerably among these studies and that our comparisons required rather conservative statistical tests.

We conclude that expectancy accounts for sequential priming in many laboratory studies (P. M. Blough, 1989, 1991, 1992; Bond \& Riley, 1991; Pietrewicz \& Kamil, 1979); in all of these, trials were separated by more than 1 sec. Nevertheless, our design may have missed extremely transient sources of facilitation. For example, Bond (1983) proposed that prey repetition, if the encounters occurred within approximately $0.4 \mathrm{sec}$, triggered an attentional mode specific to that prey. Bond's 
model would not account for longer lasting sequential effects, but the suggested mechanism might contribute during rapid prey encounters within pigeon foraging bouts.

\section{REFERENCES}

Beller, H. K. (1971). Priming: Effects of advance information on matching. Journal of Experimental Psychology, 57, 1976-1982.

Blough, D. S. (1985). Discrimination of letters and random dot patterns by pigeons and humans. Journal of Experimental Psychology: Animal Behavior Processes, 11, 261-280.

Blough, D. S. (1993). Effects on search speed of the probability of target distractor combinations. Journal of Experimental Psychology: Animal Behavior Processes, 19, 231-243.

Blough, P. M. (1984). Visual search in pigeons: Effects of memory set size and display variables. Perception \& Psychophysics, 35, 344352.

Blough, P. M. (1989). Attentional priming and visual search in pigeons. Journal of Experimental Psychology: Animal Behavior Processes, 15, 358-365.

Blough, P. M. (1991). Selective attention and search images in pigeons. Journal of Experimental Psychology: Animal Behavior Processes, 17, 292-298.

Blough, P. M. (1992). Detectability and choice during visual search: Joint effects of sequential priming and discriminability. Animal Learning \& Behavior, 20, 293-300.

Bond, A. B. (1983). Visual search and selection of natural stimuli in the pigeon: The attention threshold hypothesis. Journal of Experimental Psychology: Animal Behavior Processes, 9, 292-306.

Bond, A. B., \& RILEY, D. A. (1991). Searching image in the pigeon: A test of three hypothetical mechanisms. Ethology, 87, 203-224.

Collyer, C. E., \& Enns, J. T. (1987). Analysis of variance: The basic designs. Chicago: Nelson-Hall.

Eriksen, C. W., \& Hoffman, J. E. (1972). Some characteristics of selective attention in visual perception determined by vocal reaction time. Perception \& Psychophysics, 11, 169-171.
GuILFord, T., \& Dawkins, M. S. (1987). Search images not proven: A reappraisal of recent evidence. Animal Behavior, 35, 1838-1845. KIRBY, N. (1976). Sequential effects in an eight-choice serial reaction time task using compatible and incompatible stimulus-response arrangements. Acta Psychologica, 40, 207-216.

KIRBy, N. (1980). Sequential effects in choice reaction times. In A. T. Welford (Ed.), Reaction times (pp. 129-172). New York: Academic Press.

LAmbert, A., \& Hockey, R. (1986). Selective attention and performance with a multidimensional visual display. Journal of Experimental Psychology: Human Perception \& Performance, 12, 484495.

Miller, J. O. (1988). Components of the location probability effect in visual search tasks. Journal of Experimental Psychology: Human Perception \& Performance, 14, 453-471.

Pietrewicz, A. T., \& Kamil, A. C. (1979). Search image formation in the blue jay (Cyanocitta cristata). Science, 22, 1332-1333.

Posner, M. I., \& SNyder, C. R. R. (1975). Facilitation and inhibition in the processing of signals. In P. M. Rabbitt \& S. Dornic (Eds.), Attention and performance $V$ (pp. 669-682). San Diego, CA: Academic Press.

ReID, P. J., \& Shettleworth, S. J. (1992). Detection of cryptic prey: Searching image or search rate? Journal of Experimental Psychology: Animal Behavior Processes, 18, 273-286.

Shaw, M. L., \& SHAw, P. (1977). Optimal allocation of cognitive resources to a spatial location. Journal of Experimental Psychology: Human Perception \& Performance, 3, 201-211.

TINBERGEN, L. (1960). The natural control of insects in pinewoods: I. Factors influencing the density of predation by songbirds. Archives Neerlandaises de Zoologie, 13, 256-343.

Tsal, Y., \& LAVIE, N. (1993). Location dominance in attending to color and shape. Journal of Experimental Psychology: Human Perception \& Performance, 19, 131-139.

(Manuscript received July 27, 1993; revision accepted for publication November 17, 1993.) 